\title{
Nano-enabled immunomodulation
}

\author{
Flexibly designed nanomaterials can trigger specific immune responses and might offer promising alternatives to \\ traditional immunosuppressive therapies, cancer immunotherapies and vaccine formulations.
}

m mmunomodulation, that is tuning how the immune system responds to exogenous and endogenous danger stimuli, represents a bubbling field of research in nanomedicine. The complexity under which the immune system operates, however, is staggering. An intricate network of cell types and subtypes direct the concerted actions of the innate and adaptive arms of the immune system. They coordinate, through different signalling molecules, the response against danger signals, and curb, in healthy conditions, the abnormal reaction against self-antigens that arise in autoimmune diseases.

The possibilities for nano-enabled immunomodulation are therefore multiple, and so are the challenges. This Focus issue illustrates a few examples.

The Review by Cifuentes-Rius and co-workers, focuses on nanotechnology approaches that target dendritic cells (DCs) to induce immune tolerance. Being the point of contact between the innate and the adaptive immune system, DCs are a heterogeneous class of antigen-presenting cells that play a prominent role in maintaining the delicate balance between immunity and tolerance. Tolerogenic immunotherapies seek to induce self-tolerance through DC reprogramming. Traditionally this is achieved ex vivo in cells isolated from patients, which are first cultured in a medium that induces a tolerogenic phenotype and subsequently reinfused into the body. Rationally engineered nanoparticles might instead allow DC reprogramming in vivo. The authors describe the different routes used to generate tolerogenic responses in animal models, and their possible clinical applications in organ transplants and the treatment of diabetes mellitus and multiple sclerosis, with the challenges associated.

$\mathrm{T}$ cells are another major class of immune cells amenable to engineering approaches, with particular relevance for cancer immunotherapy. Like DCs, they can be extracted from a patient's blood, modified, expanded and reinfused into the body to kill tumour cells, in a process called adoptive T-cell therapy (ACT).

ACTs hold tremendous translational potential, as demonstrated by the US Food and Drug Administration approval of three chimeric antigen receptor (CAR) T-cell therapies in 2017 and $2020^{1-3}$. However several bottlenecks hamper their wide implementation, such as insufficient $\mathrm{T}$ cell expansion in vitro, poor trafficking to the target tumours, $T$ cell exhaustion in the tumour site and loss of targeting antigens on cancer cells. In their Review, Gong and colleagues discuss the solutions that nanomedicine offers to overcome such issues, the advantages it presents for the potential application of ACTs to treat solid tumours, and the outstanding questions that remain for its clinical application in $\mathrm{T}$ cell immunotherapy.

The challenge of translating immuno-oncology nanomedicine results from the preclinical to the clinical setting is the subject of the Perspective by Jiang and co-workers. Nanomaterials can improve the outcome of immunotherapies while reducing their side effects but their success eventually relies on the tumoricidal capabilities of the immune system. Therefore, when designing preclinical experiments, it is crucial to consider the different variables that might influence the immune system responses, in order to align preclinical results with clinical outcomes. Tumour molecular heterogeneity, microbiota, sex disparities, immunosenescence, pseudoprogression and systemic toxicity, are all confounding elements that might hinder the advancement of nano-immuno medicine in the clinic. Paying closer attention to the animal models used for the preclinical studies, improving condition reporting and expanding toxicity analyses are some of the suggestions proposed by the authors to account for these factors.

Moving away from therapy, the Review by Singh provides an overview of how nanotechnology may help vaccine development against infectious diseases. Nanovaccines can overcome the barrier to enter the lymphatic system and reach, via different mechanisms, the $\mathrm{B}$ cell follicles of the lymph nodes. There they can present their antigens to B cells, promoting their maturation and eventual differentiation into long-lived antibody secreting plasma $B$ cells and memory B cells, which protect from re-infection. The article reviews the bioengineering strategies that produced efficient nanovaccines in animal models, and analyses the current challenges in the field, that is, the effect of the microbiome in nanovaccine efficacy; the potential antibody-dependent exacerbation of the disease due to suboptimal antibody response; how to design nanovaccines that elicit broadly neutralizing antibodies for HIV and influenza; how to account for virus mutations; and how to scale up nanovaccine manufacturing.

This special issue showcases only some of the ongoing research in the field of nano-immunoengineering. Aside from the multiple strategies developed to improve cancer immunotherapies, chronic inflammatory diseases such as atherosclerosis can also benefit from nanoimmunotherapy approaches ${ }^{4}$. Moreover, biomimetic strategies using nanoparticles coated with leukocyte-derived membranes have recently been applied in preclinical models to ameliorate severe inflammatory conditions ${ }^{5}$. Finally, nanomaterials, with their uniquely tuneable features and the possibility of organising surface epitopes in specific spatial arrangements, can be used to explore basic aspects of immunology, offering insights on how the immune system recognises specific danger signals on pathogens, and on how immune cells interact between each other ${ }^{6}$. These results will feed, in turn, future applicative bioengineering efforts.

Published online: 12 January 2021 https://doi.org/10.1038/s41565-020-00842-8

\footnotetext{
References

1. FDA approval brings first gene therapy to the United States. FDA https://www.fda.gov/news-events/press-announcements/ da-approval-brings-first-gene-therapy-united-states (2017).

2. FDA approves CAR-T cell therapy to treat adults with certain types of large B-cell lymphoma. FDA https://www.fda.gov/ news-events/press-announcements/fda-approves-car-t-celltherapy-treat-adults-certain-types-large-b-cell-lymphoma (2017).

3. U.S. FDA approves Kite's Tecartus, the first and only CAR T treatment for relapsed or refractory mantle cell lymphoma. Business Wire https://www.businesswire.com/news/home/ 20200724005428/en/ (2020).

4. Duivenvoorden, R. et al. Nat Rev. Cardiol. 16, 21-32 (2019).

5. Sushnitha, M., Evangelopoulos, M. Tasciotti, E. \& Taraballi, F. Front. Bioeng. Biotechnol. https://doi.org/10.3389/fbioe. 2020.00627 (2020).

6. Hubbell, J., Thomas, S. \& Swartz, M. Nature 462, 449-460 (2009).
} 